\title{
BMJ Open Prevalence and outcomes of multimorbidity in South Asia: a systematic review
}

Sanghamitra Pati, ${ }^{1}$ Subhashisa Swain, ${ }^{1}$ Mohammad Akhtar Hussain, ${ }^{2}$
Marjan van den Akker, ${ }^{3,4}$ Job Metsemakers, ${ }^{3}$ J André Knottnerus, ${ }^{3}$ Chris Salisbury ${ }^{5}$

To cite: Pati S, Swain S, Hussain MA, et al. Prevalence and outcomes of multimorbidity in South Asia: a systematic review. BMJ Open 2015;5:e007235. doi:10.1136/bmjopen-2014007235

- Prepublication history and additional material is available. To view please visit the journal (http://dx.doi.org/ 10.1136/bmjopen-2014007235).

Received 21 November 2014 Revised 8 July 2015 Accepted 19 July 2015

For numbered affiliations see end of article.

Correspondence to Dr Sanghamitra Pati; sanghamitra.pati@iiphb.org

\section{ABSTRACT}

Objective: To systematically review the studies of prevalence, patterns and consequences of multimorbidity reported from South Asia.

Design: Systematic review.

Setting: South Asia.

Data sources: Articles were retrieved from two electronic databases (PubMed and Embase) and from the relevant references lists. Methodical data extraction according to Preferred Reporting Items for Systematic reviews and Meta-Analyses (PRISMA) guidelines was followed. English-language studies published between 2000 and March 2015 were included.

Eligibility criteria: Studies addressing prevalence, consequences and patterns of multimorbidity in South Asia. Articles documenting presence of two or more chronic conditions were included in the review. The quality and risk of bias were assessed using STROBE criteria.

Data selection: Two reviewers independently assessed studies for eligibility, extracted data and assessed study quality. Due to heterogeneity in methodologies among reported studies, only narrative synthesis of the results was carried out.

Results: Of 11 132, 61 abstracts were selected and 13 were included for final data synthesis. The number of health conditions analysed per study varied from 7 to 22 , with prevalence of multimorbidity from $4.5 \%$ to $83 \%$. The leading chronic conditions were hypertension, arthritis, diabetes, cardiac problems and skin diseases. The most frequently reported outcomes were increased healthcare utilisation, lowered physical functioning and quality of life, and psychological distress.

Conclusions: Our study, a comprehensive mapping of multimorbidity research in South Asia, reveals the insufficient volume of work carried out in this domain. The published studies are inadequate to provide an indication of the magnitude of multimorbidity in these countries. Research into clinical and epidemiological aspects of multimorbidity is warranted to build up scientific evidence in this geographic region. The wide heterogeneity observed in the present review calls for greater methodological rigour while conducting these epidemiological studies.

Trial registration number: CRD42013005456.

\section{INTRODUCTION}

In the past few decades, chronic diseases have replaced infectious diseases and
Strengths and limitations of this study

- Our systematic review identifies a large knowledge gap in the epidemiology of multimorbidity in South Asia, where few studies have been conducted to investigate multimorbidity.

- Our review is the first to undertake a comprehensive mapping of multimorbidity studies in South Asia and demonstrates the need for systematic enquiries on multimorbidity to be undertaken in this region.

- Since multimorbidity is not well indexed in literature databases, we might have inadvertently omitted some studies.

- A quantitative synthesis could not be performed due to a large amount of heterogeneity among the selected studies.

assumed the dominant healthcare burden. ${ }^{1}$ Coexistence of multiple chronic diseases in a single individual, known as multimorbidity, is increasingly becoming the norm. ${ }^{2}$ Individuals with multimorbidity register higher mortality rates, incur increased healthcare expenditure, are frequently hospitalised, and experience disturbed physical and mental health, affecting overall functioning and quality of life. ${ }^{3} 4$ Owing to its negative consequences and high resource use associated, multimorbidity has attracted considerable interest and attention among clinicians and public health researchers alike. ${ }^{5}$ A considerable corpus of primary care research over the last decades has been performed around this area, in developed countries. $^{6-9}$ Prevalence estimates in these countries have shown varying figures ranging from $39.5 \%$ in Spain to $13 \%$ in the Netherlands. ${ }^{10} 11$ A study involving primary care patients in Scotland has revealed one quarter of patients to have multimorbidity, with one-third of them being young. ${ }^{12}$ This study strongly urged the global health community to be adequately prepared to be responsive to the challenges of multimorbidity. Nonetheless, the population-based studies 


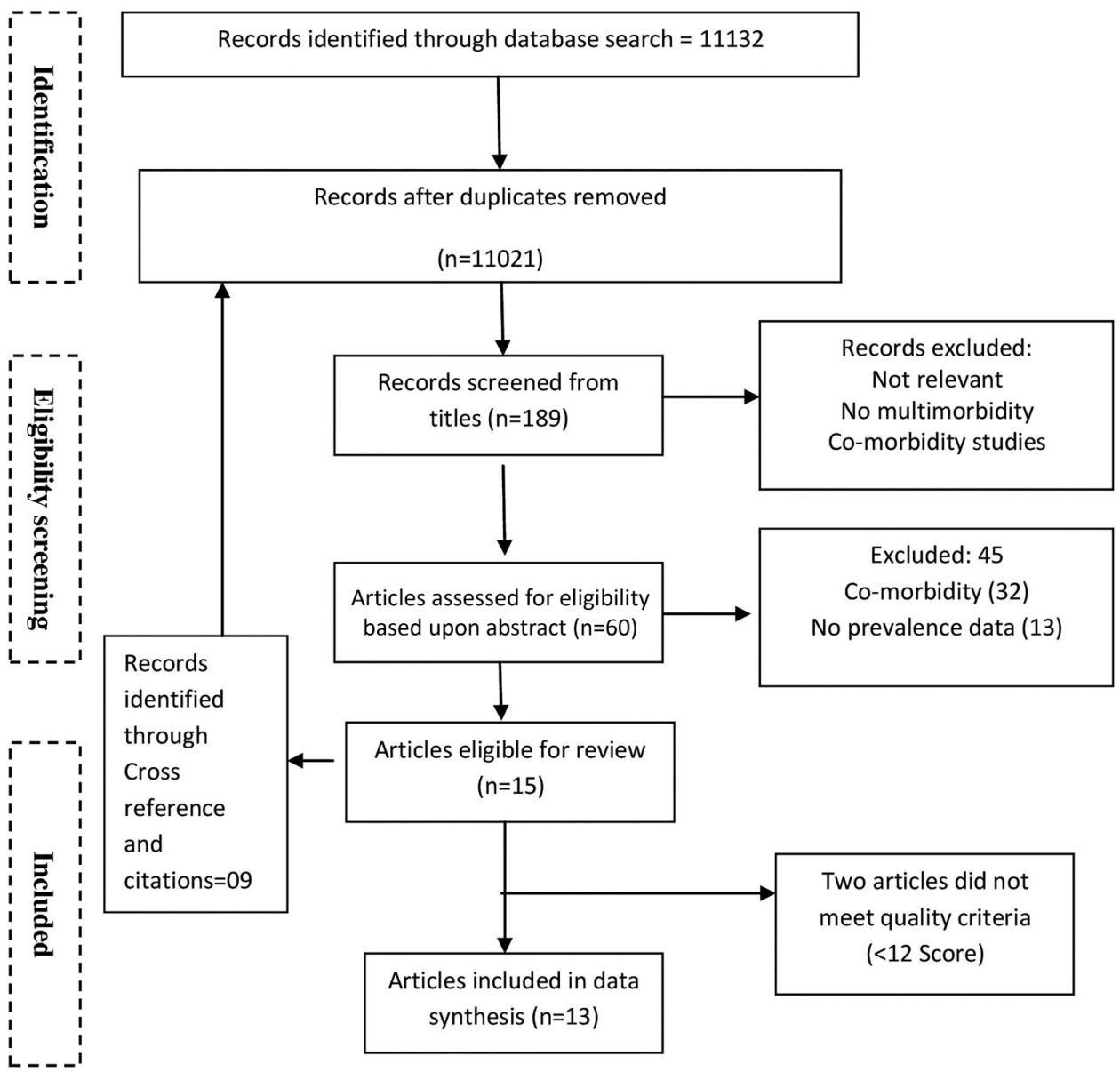

Figure 1 PRISMA (Preferred Reporting Items for Systematic reviews and Meta-Analyses) flowchart of the literature search.

from several middle income countries such as Ghana, Brazil and South Africa reported prevalence of multimorbidity as high as $38.5 \% .^{13-15}$ However, to date, the majority of research from low- and middle-income countries (LMICs) are focused on a single or specific illness, or on the coexistence of a relatively small number of diseases such as cardiovascular ailments, diabetes and cancer, and the presence of unrelated or incongruent multiple chronic conditions has not been investigated in detail. $^{16} 17$

Together, home to approximately one-fifth of the world's population, South Asia deserves special attention in the context of multimorbidity. All of the seven countries in this region are LMICs. ${ }^{18}$ Along with rapid urbanisation and demographic transition, these countries are now experiencing a shift from communicable to noncommunicable diseases, and multimorbidity could be an emergent phenomenon. Given the high younger population, the projected magnitude can be enormous, and the extant unprepared health system and limited resources could cumulatively add to the adverse impacts.

Several studies are available from individual South Asian countries on the level of selected or individual chronic diseases among the adult population. However, to the best of our knowledge, to date, there are no comprehensive systematic reviews on multimorbidity among adults residing in the South Asian region, and therefore a contextual understanding essential for developing and aligning health services to meet patient care is lacking. The present systematic review is the first attempt to landscape multimorbidity research in South Asia and to systematically evaluate published studies (longitudinal, cross sectional) documenting occurrence, pattern and consequences of multimorbidity in the adult population in South Asian countries, thus enabling comparison with other regions. It is expected that the information acquired would identify existing knowledge gaps and guide future research needs into multimorbidity in this region. The specific objectives were to (1) estimate the prevalence of multimorbidity, and (2) study the patterns of occurrence and its consequences in South Asia. The focus of the review was limited to multimorbidity defined as the co-occurrence of multiple chronic diseases in the same individual or mean disease count per individual.

\section{METHODS}

A systematic review of published studies reporting multimorbidity among adults residing in South Asia was undertaken in accordance with the Preferred Reporting Items for Systematic reviews and Meta-Analyses 
Table 1 Studies reporting prevalence of multimorbidity in South Asia

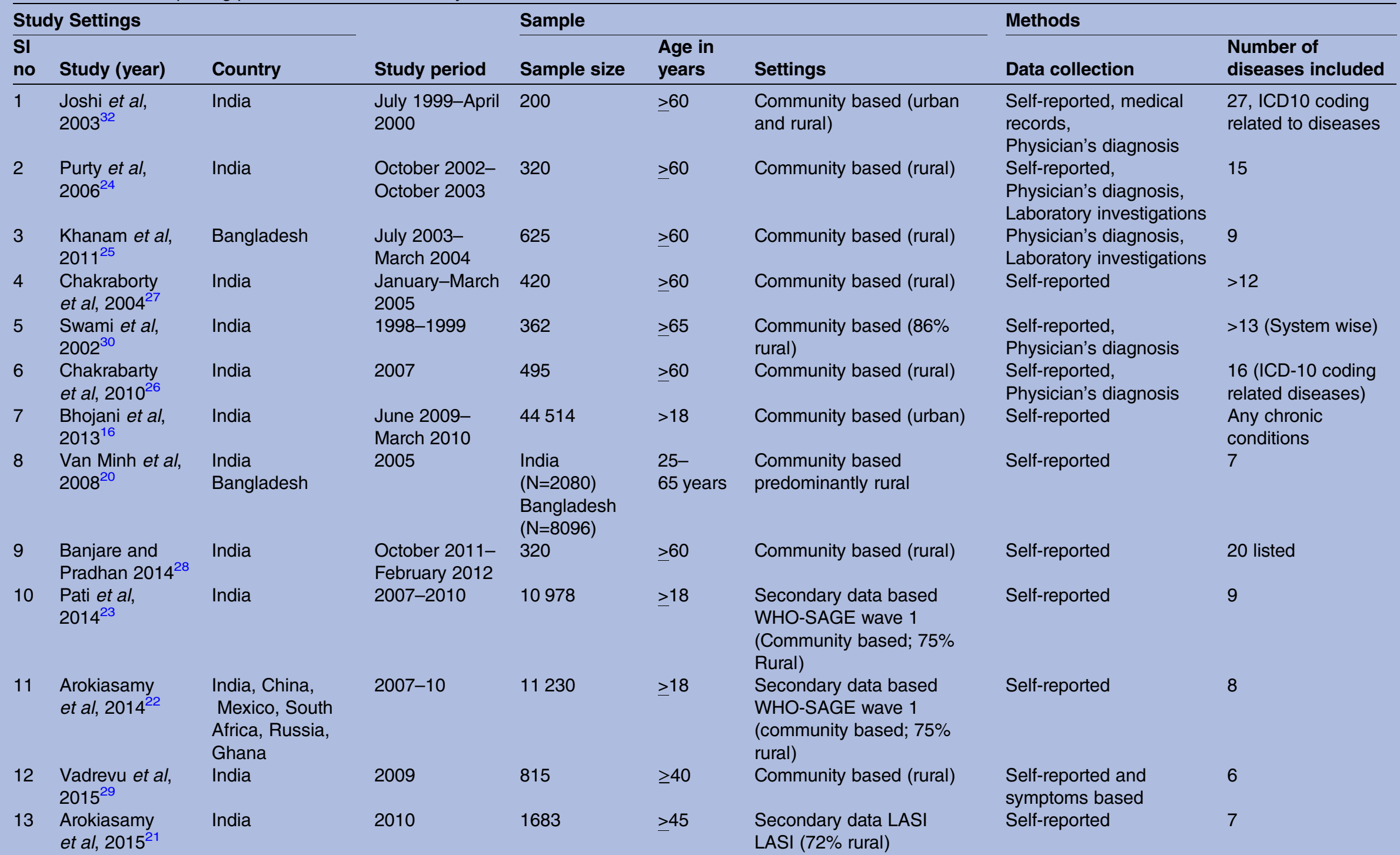

ICD10, 10th revision of the International Statistical Classification of Diseases and Related Health Problems; LASI, Longitudinal Aging Study India; WHO-SAGE, World Health OrganizationStudy on global AGEing and adult health. 
(PRISMA) statement. The methodology has been published in PROSPERO with registration ID: CRD42013005456. (http://www.crd.york.ac.uk/PROSPERO/).

\section{Inclusion and exclusion criteria}

Selection of articles was based on following inclusion criteria, they were: (1) original studies documenting prevalence, patient factors associated with multimorbidity and consequences of it; or (2) studies reporting results that allowed calculation of prevalence; (3) studies having participants of more than 18 years of age; (4) conducted either in a primary care/outpatient setting or general population from the above mentioned South Asian countries; (5) studies that had published results between 1990 and March 2015. As multimorbidity first came to prominence in the early 1990s, we included articles published in the English language between 1 January 1990 and 31 March 2015.

For those studies in which multimorbidity was not defined, we made an operational definition of 'studies documenting two or more chronic conditions, even though not mentioning the term multimorbidity'. These were also included for data synthesis. Any study that began with a preliminary selection of index disease (studies of comorbidity) was excluded.

\section{Search strategy for identification of articles}

We systematically explored Pub Med and EMBASE electronic databases, and Google Scholar search engines, to locate the relevant articles. We categorised the search terms according to location, methodology and outcomes: (1) Location: 'India, Pakistan, Nepal, Bhutan, Bangladesh, Sri Lanka, Maldives, South Asia'. ${ }^{18}$ (2) Method: 'prevalence, epidemiology, cluster, pattern'. (3) Outcome: 'multimorbidity, multimorbid, multi-morbidity, multiple conditions, co-morbid, multiple diseases, multiple chronic diseases, multiple chronic conditions, multiple illnesses, multiple diagnoses, multi-pathology, chronic condition, chronic diseases'. The 'AND' Boolean operator was used to combine search terms across the categories and 'OR' was used to combine within the categories. To broaden the scope of our research, we also applied the linguistic variations of multimorbidity in the search strategy. Further, we limited the search to those studies that only involved human participants, had abstracts available and were published between 1 January 1990 and 31 March 2015. To obtain additional publications, reference lists of retrieved articles were hand searched using snowballing techniques. Wherever possible, forward citations of the studies retrieved during the literature search were traced and screened for possible inclusion. Furthermore, search of relevant websites, namely multimorbidity research network of university of Sherbrook (http://crmcspl-blog.recherche.usherbrooke.ca/) and WHO (http://www.who.int/en/), was also performed. A summary of the search strategy adopted for the review is outlined (see online supplementary appendix 1).

\section{Data management}

First, all hits obtained were gathered and duplicates removed. Potentially relevant articles were selected through initial title and abstract screening by two authors (MAH and SS) independently. In the next step, the full text copies of these relevant articles were retrieved. We retained those articles that studied the prevalence of more than two chronic conditions without any index disease, even if they were not using the term 'multimorbidity'. Articles meeting all inclusion criteria were retained for quality assessment and data extraction. For data extraction, a special form was constructed. Two authors (MAH and SS) independently assessed each of these 61 retrieved articles for inclusion, extracted data and cross checked data extraction forms. Any discrepancies regarding eligibility between the two reviewers were resolved by consensus with two other authors (SP, CS).

\section{Assessment of study quality}

Two authors (MAH and SS) independently assessed risk of bias and study quality, using standard 'strengthening the reporting of observational studies in epidemiology' (STROBE) checklist. $^{9}{ }^{19}$ Any disagreement arising on quality was sorted out in consultation with two other authors (SP and CS). For observational study designs, risk of bias was assessed for three domains: selection bias, information bias (differential misclassification and non-differential misclassification) and confounding. Risk of bias for each domain was assessed as either 'Yes' or 'No'. Studies that had a risk of bias in each domain, including a risk of confounding, were classified as having more of a risk of bias. Each reviewer independently determined a global quality score for each article, giving one point for each STROBE item the article addressed. To be retained in our review, articles had to have a quality score of at least 12 of a possible 23 .

\section{Data extraction}

For each included study, we extracted the following information: (1) authors and publication year; (2) title and journal; (3) study country and location (urban or rural); (4) study design; (5) sampling strategy (random or non-random); (6) sample size; (7) sample characteristics such as age and gender; (8) number of conditions included; (9) definition of multimorbidity considered; (10) prevalence (overall and gender- or locationspecific) of multimorbidity; (11) consequences of multimorbidity in terms of health-related quality of life (HRQoL), functional status, healthcare utilisation and healthcare expenditure (objective or subjective); and (12) risk factors significantly associated with multimorbidity.

We decided not to perform meta-analysis as we judged that the included studies were heterogeneous in different aspects, including: populations (different ages and settings), variable definitions (including different definitions of exposures and outcomes) and analytical strategies (adjustment for different confounders). 
Table 2 Characteristics of selected studies concerning prevalence of multimorbidity and risk factors

\begin{tabular}{|c|c|c|c|c|c|c|}
\hline $\begin{array}{l}\text { Author, year of } \\
\text { publication }\end{array}$ & Country & $\begin{array}{l}\text { Use of term } \\
\text { multimorbidity } \\
\text { in the study }\end{array}$ & $\begin{array}{l}\text { Definition of } \\
\text { multimorbidity }\end{array}$ & $\begin{array}{l}\text { Results } \\
\text { Prevalence }\end{array}$ & Risk factors & Consequences \\
\hline Joshi 2003 & India & No & Not described & $83 \% *$ & Not described & No \\
\hline Purty 2006 & India & No & Not described & $24.1 \%$ & Not described & No \\
\hline Khanam 2011 & Bangladesh & Yes & $\begin{array}{l}\text { Two or more chronic } \\
\text { medical conditions }\end{array}$ & $53.8 \%$ & $\begin{array}{l}\text { Women (OR 3.32; } 1.88-5.86 \text { ) } \\
\text { High-income group } \\
\text { (OR 1.93; 1.14-3.27) }\end{array}$ & No \\
\hline Chakraborty 2004 & India & No & Not described & $54.4 \%$ & & No \\
\hline Swami 2002 & India & No & Not described & $69.9 \%$ & $\begin{array}{l}\text { Urban } \\
\text { Female }\end{array}$ & No \\
\hline Chakrabarty 2010 & India & No & Not described & $53.7 \%$ & Not described & No \\
\hline Bhojani 2013 & India & No & Not described & $4.5 \%$ & Not described & No \\
\hline Van Minh 2008 & $\begin{array}{l}\text { India } \\
\text { Bangladesh }\end{array}$ & No & Not described & $\begin{array}{l}\text { India }=5.86 \% \\
\text { Bangladesh }=10.75 \%\end{array}$ & Not described & No \\
\hline Banjare 2014 & India & Yes & $\begin{array}{l}\text { Presence of two or more } \\
\text { chronic diseases }\end{array}$ & $56.5 \%$ & 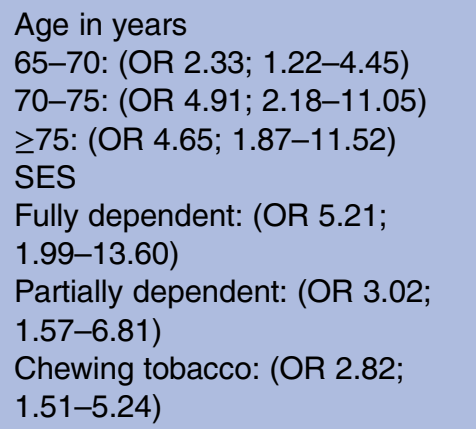 & No \\
\hline Pati 2014 & India & Yes & $\begin{array}{l}\text { Presence of two or more } \\
\text { chronic diseases }\end{array}$ & $8.9 \%$ & $\begin{array}{l}\text { Age in years } \\
\text { 30-39: (OR 4.11; 2.18-7.74) } \\
\text { 40-49: (OR 7.87; 4.25-14.59) } \\
\text { 50-59: (OR 16.15; 8.83-29.54) } \\
\text { 60-69: (OR 23.56; 13.08-42.44) } \\
\text { >70: (OR 39.15; 20.72-73.98) }\end{array}$ & $\begin{array}{l}\text { Increase healthcare } \\
\text { utilisation and } \\
\text { expenditure }\end{array}$ \\
\hline Arokiasamy 2014 & India & Yes & $\begin{array}{l}\text { Simultaneous presence of } \\
\text { two or more chronic } \\
\text { diseases }\end{array}$ & $20.8 \%$ & $\begin{array}{l}\text { Age in years } \\
\text { 50-59: (OR } 3.12 ; 2.88-3.37) \\
\text { 60-69: (OR 5.24; 4.83-5.7) } \\
\geq 70:(\text { OR } 7.53 ; 6.9-8.26) \\
\text { Widow/er (OR } 1.45 ; 1.26-1.64 \text { ) } \\
\text { Obese (OR 1.59; 1.48-1.71) } \\
\text { High risk WHR } \\
\text { (OR 1.25; } 1.17-1.32 \text { ) } \\
\text { Inactive PA (OR } \\
\text { 1.13; } 1.07-1.19)\end{array}$ & $\begin{array}{l}\text { Poor self-rated health } \\
(\mathrm{SRH}) \\
\text { Increased functional } \\
\text { limitation } \\
\text { Poor quality of life } \\
\text { Depression }\end{array}$ \\
\hline
\end{tabular}

Author, year of

Purty 2006

India

Two or more chronic

$69.9 \%$

$53.7 \%$

India $=5.86 \%$

Bangladesh $=10.75 \%$

Presence of two or more chronic diseases

$1.13 ; 1.07-1.19)$ 


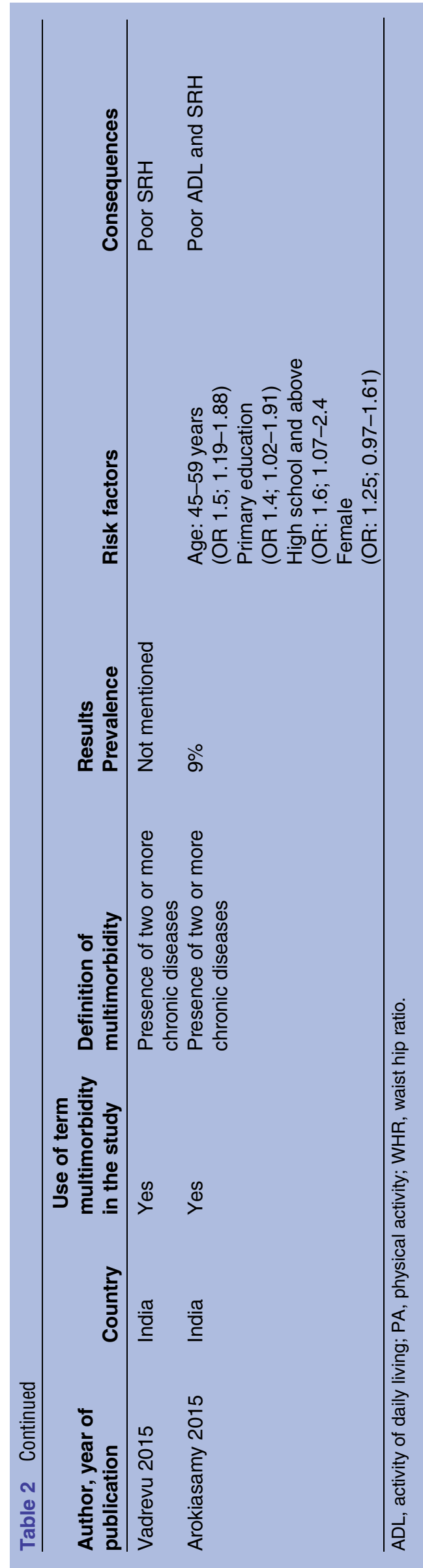

RESULTS

\section{Yield of search strategy}

The searches mentioned above yielded 11132 articles. After discarding duplicates, 11021 were selected for title screening. Careful screening of titles identified 189 articles for abstract reading, from which 61 were shortlisted for full text review. Finally, 13 articles were included for this systematic review. Reasons for exclusion of the remaining articles are indicated in figure 1.

\section{Study characteristics and quality}

The key characteristics of the studies are presented in table 1. All the studies were from India and Bangladesh. Four studies were carried out on a nationally representative sample and the rest adopted ad hoc study designs. ${ }^{20-23}$ All were cross-sectional and quantitative in nature. All studies were community based. Six studies recruited participants exclusively from rural areas, ${ }^{24-29}$ two from urban area ${ }^{16}{ }^{30}$ and five included urban as well as rural participants. ${ }^{20-23} 31$ The sample size of the included studies varied from 90 to 44514 , and included males as well as females. Seven studies exclusively included participants over 60 years of age. ${ }^{24-28} \quad 3032$ Proportion estimation was the most frequently used statistical measure. On a quality assessment scale, five studies scored between 12 and $18^{24} 262729$ 30; whereas eight studies scored more than $18,{ }^{16} \quad 20-23 \quad 25 \quad 2832$ and five articles scored between 12 and 18 (see online supplementary appendix 2).

\section{Definition and estimation of multimorbidity}

'Multimorbidity' was defined and used in six studies (table 2). ${ }^{21-2325} 2829$ The remaining seven articles mentioned the presence of two or more chronic conditions without using the term 'multimorbidity'. Twelve used a predefined list of chronic conditions ranging from 7 to 16, (see online supplementary appendix 3) and one adopted a free listing method. ${ }^{16}$ For identification of patients with chronic conditions, different approaches were used, namely, self-reports in five studies, ${ }^{20} 21232728$ self-reports and physician diagnosis in four studies, ${ }^{24} 263032$ and, in other studies, a combination of physician's diagnosis and laboratory investigations, ${ }^{25}$ and both self-reported and symptom based approaches were used. ${ }^{22}{ }^{29}$ International classification for disease coding was used in three studies and the remaining used arbitrary systems of coding. ${ }^{26} 32$

Five studies had stated the objective of estimating the prevalence of multimorbidity. ${ }^{21-23} 2829$ The rest were intended to identify multiple chronic conditions. The prevalence of multimorbidity varied from $4.5 \%{ }^{16}$ to $83 \% .^{32}$ Among the population aged 60 years or over, the prevalence ranged from $24.1 \%{ }^{24}$ to $83 \%,{ }^{32}$ while for the remaining adult population it was from $4.5 \%{ }^{16}$ to $20.8 \% .{ }^{21}$ Prevalence of multimorbidity among studies adopting self-reported methodology ranged from $4.3 \%{ }^{20}$ to $56.8 \%{ }^{16}$ Among studies from national representative samples, the prevalence varied from $4.3 \%^{20}$ to $8.9 \% .^{23}$ 
The only study using physician diagnosis and laboratory investigations reported multimorbidity prevalence of $53.8 \% .^{25}$ The prevalence varied from $24.1 \%^{24}$ to $83 \%{ }^{32}$ among studies that used both a self-reported and clinical examination approach. All the studies had followed a simple count method adding up the number of chronic diseases.

\section{Patterns, correlates and consequences of multimorbidity}

The leading chronic conditions reported were hypertension, arthritis, diabetes, cardiac problems and skin diseases (see online supplementary appendix 3). Apart from one,${ }^{28}$ no other studies reported the pattern of diseases or commonly occurring disease clusters. The most frequently reported consequences were increase in healthcare utilisation, ${ }^{23} 27{ }^{30}$ lowering of physical functioning, ${ }^{21}{ }^{22}$ disability, ${ }^{32}$ quality of life, ${ }^{21}$ healthcare expenditure $^{23}$ and psychological distress. ${ }^{32}$ Only one study $^{21}$ explored the morbidity burden or severity and HRQoL. Four studies ${ }^{21-23} 28$ identified age to be strongly associated with multimorbidity. Two studies ${ }^{22} 28$ considered risk factors such as tobacco use, obesity, waist hip ratio and physical activity, for prediction of multimorbidity. Three studies ${ }^{21} 22{ }^{29}$ looked at the impact of multimorbidity on self-rated health. One study ${ }^{23}$ explored the effect of multimorbidity on healthcare utilisation and expenditure. Positive association between multimorbidity and depression was reported in two articles. ${ }^{22} 32$

\section{DISCUSSION}

The present systematic review intended to summarise the scientific evidence accumulated in the past two decades pertaining to multimorbidity in South Asia. We identified only 13 studies, confined to two countries. Earlier reviews by Western authors also noted the limited representation of developing countries in multimorbidity research. ${ }^{9}$ South Asians have already been shown to be an inherently high-risk group for developing cardiometabolic and other chronic diseases, and thus multimorbidity may be significantly prevalent in these populations. ${ }^{33}$ Nevertheless, the scarcity of publications in our review demonstrates an obvious mismatch between the need for work versus work accomplished in this area.

Five studies had the primary objective of estimating multimorbidity, while for others, it was a secondary observation, which further reduces the strength of evidence on this topic. Interestingly, six studies have assessed the prevalence of two or more chronic conditions without citing the term 'multimorbidity', suggesting low familiarity of the researchers with this entity. Five were published in the year 2014-2015, indicating the recent growing interest in multimorbidity in this region. At the same time, it also suggests the continuing foothold of single disease and infectious conditions among South Asian health system researchers.
The wide variance in prevalence estimates observed in our review stems from the diversities in study methodologies. For instance, sample size estimation, age group of the study participants, and inclusion and exclusion criteria, differed considerably between studies, which makes comparability difficult. Similar heterogeneity was observed in a review documenting prevalence of comorbidities in Australia, where diverse methods and study settings were the contributing reasons. ${ }^{34}$ Another review on multimorbidity patterns also exhibited considerable methodological variability in terms of sample size, age and recruitment of study participants, data source and number of base line diseases. ${ }^{35}$ Four of the 13 studies used secondary data from national surveys. ${ }^{20-23}$ None of the reported studies had the intention or objective of looking at multimorbidity, per se.

Overall quality assessment revealed major lacunae in methodological aspects, which included ascertainment and case definition of multimorbidity, selection of source population, and inclusion and exclusion criteria. Even though some of these weaknesses were noted by the researcher in the limitations section, none of the studies tried to address bias. The wide heterogeneity observed due to non-uniformity in methodology and disease screening criteria makes comparability difficult and explains to some extent the large diversity observed. Owing to the inherent biases in the original studies' estimation, quantification of the prevalence could not be assessed.

The majority of the authors did not describe the criteria for selection of chronic diseases. Where they did, the most common were those conditions with a high prevalence and/or clinical relevance. As the number and type of conditions included determines multimorbidity estimation, the reported prevalence in these studies may not be reflective of the real burden. Moreover, there was ambiguity in disease definitions, such as doubt over whether ischaemic heart disease and myocardial infarction should be considered separate entities. Thus, efforts should be first directed at preparing a panel of chronic diseases with standardised definitions of each condition. This would help in minimising the inter-study variations, reduce possible selection bias of specific chronic diseases, and result in more reliable and comparable estimation of multimorbidity. Further, none of the included studies were undertaken in a primary care setting, which constitutes an important knowledge gap and substantiates the earlier evidence of non-availability exploring multimorbidity in primary care settings in LMICs. ${ }^{35}$ In view of the integral role of primary care in the management of patients with longterm conditions, ${ }^{6}$ and primary care being the major healthcare provider for the population in this region, ${ }^{36}$ future studies should include these practices in exploring multimorbidity.

The study populations in most articles were aged 60 years or above, which might have introduced an element of age bias. One possible reason could be that most researchers have assumed multiple chronic 
conditions to be more akin to the geriatric population. Multimorbidity is not limited to old age alone, as it is significantly prevalent among the young population as well. ${ }^{12}$ Equating multimorbidity with ageing could underestimate its real magnitude. This has important implications, especially for South Asian countries, as the majority of this region's population is young and possesses the risk of escalation of burden of multiple chronic conditions in the future.

Many authors have emphasised the importance of examining the pattern of multimorbidity in addition to quantifying the conditions. Identification of high frequency clusters is important for developing specific treatment guidelines and better patient management. However, only one study in our review explored the clustering of diseases. ${ }^{28}$ The recent review on pattern of associative multimorbidity by Prados-Torres et $a l^{35}$ reflected similar findings with lack of published literature from LMICs.

The negative health consequences of multimorbidity have placed it in the forefront of healthcare and research, the most relevant sequelae being increased healthcare utilisation, decreasing HRQoL, impaired physical functioning, poor mental health and increased healthcare expenditure. ${ }^{37}{ }^{38}$ In our review, less than half the studies considered this aspect by assessing physical and mental functioning and healthcare utilisation. Few have looked at the impact of multimorbidity on HRQoL and self-reported health. In view of the informative role of outcome measurement in the design of interventions, future studies investigating the burden of multimorbidity in South Asia need to embrace this dimension.

Finally, the insufficient volume of published work gathered through our review is inadequate to provide an indication of the magnitude of multimorbidity in South Asian countries. This is both surprising and concerning since multimorbidity is a well-recognised priority in chronic disease research worldwide and no longer considered exotic. Increased research into clinical and epidemiological aspects of multimorbidity is essential to build up the scientific evidence in this geographic region. More importantly, the wide heterogeneity observed in the present review insinuates the need of greater methodological rigour while conducting these epidemiological studies.

\section{Study limitations}

The major limitation of our systematic review is the difficulty in ensuring that all the relevant literature has been included. Since multimorbidity is not well indexed in literature databases we might have inadvertently omitted some studies. We tried to compensate for this by using an extended list of text words referring to the term multimorbidity as well as including any studies reporting two or more chronic conditions excluding comorbidity. Owing to the large heterogeneity among the studies, we could not perform quantitative synthesis of the prevalence estimates. An inherent limitation of any systematic review is the necessity to restrict a search period, which involves the exclusion of new studies after the end date. This might have resulted in omission of very recent studies.

\section{CONCLUSIONS AND FUTURE RESEARCH}

Multimorbidity still remains an unexplored area of research in South Asia. Despite the growing prevalence of chronic diseases, the evidence base for multimorbidity and its consequences is extremely limited for this region. Since multimorbidity is a major challenge to primary care, prevalence studies in these settings are recommended. Further, relevant outcome measures such as healthcare utilisation, quality of life, activity of daily living and healthcare expenditure should be examined in unison with prevalence. Care should be taken to adopt a uniform operational definition of multimorbidity, and an iterative list of chronic conditions contextualised for individual countries should be developed while assessing multimorbidity.

\section{Author affiliations}

${ }^{1}$ Indian Institute of Public Health, Bhubaneswar, Public Health Foundation of India, Bhubaneswar, Odisha, India

${ }^{2}$ Division of Epidemiology and Biostatistics, School of Public Health, University of Queensland, Brisbane, Queensland, Australia

${ }^{3}$ Department of Family Medicine, School CAPHRI, Maastricht University, Maastricht, The Netherlands

${ }^{4}$ Department of General Practice, KU Leuven, Leuven, Belgium

${ }^{5}$ Centre for Academic Primary Care, School of Social and Community Medicine, University of Bristol, Bristol, UK

Contributors SP, SS, MAH and CS provided the concept and designed the study. SP, MAH and SS extracted the data, and carried out the analysis and interpretation of data. SP, SS, MHA, MVdA and CS were involved in drafting the manuscript, and MVdA, JAK and JM revised it critically for important intellectual content. All the authors read and approved the final manuscript.

Funding This work was supported by a Wellcome Trust Capacity Strengthening Strategic Award to the Public Health Foundation of India and a consortium of UK universities. The sponsors of the study had no role in study design, data collection, data analysis, data interpretation, or writing of the report.

\section{Competing interests None declared.}

Provenance and peer review Not commissioned; externally peer reviewed.

Data sharing statement Additional data can be accessed via the Dryad data repository at http://datadryad.org/ with the doi:10.5061/dryad.n895b.

Open Access This is an Open Access article distributed in accordance with the terms of the Creative Commons Attribution (CC BY 4.0) license, which permits others to distribute, remix, adapt and build upon this work, for commercial use, provided the original work is properly cited. See: http:// creativecommons.org/licenses/by/4.0/

\section{REFERENCES}

1. Alwan A. World Health Organization. Global status report on noncommunicable diseases 2010. Geneva, Switzerland: World Health Organization, 2011.

2. Starfield B, Kinder K. Multimorbidity and its measurement. Health Policy 2011;103:3-8.

3. van den Akker M, Buntiux F, Metsemakers JF, et al. Multimorbidity in general practice: prevalence, incidence and its determinants of co-occurring chronic and recurrent diseases. J Clin Epidemiol 1998;51:367-75.

4. Fortin $\mathrm{M}$, Soubhi $\mathrm{H}$, Hudon $\mathrm{C}$, et al. Multimorbidity's many challenges. BMJ 2007;334:1016-17. 
5. Barnett K, Mercer SW, Norbury M, et al. Epidemiology of multimorbidity and implications for health care, research, and medical education: a cross-sectional study. Lancet 2012;380:37-43.

6. Laux G, Kuehlein T, Rosemann T, et al. Co- and multimorbidity patterns in primary care based on episodes of care: results from the German CONTENT project. BMC Health Serv Res 2008;8:14.

7. Mercer SW, Smith SM, Wyke S, et al. Multimorbidity in primary care: developing the research agenda. Fam Pract 2009;26:79-80.

8. van den Bussche $\mathrm{H}$, Koller D, Kolonko $\mathrm{T}$, et al. Which chronic diseases and disease combinations are specific to multimorbidity in the elderly? Results of a claims data based cross-sectional study in Germany. BMC Public Health 2011;11:101.

9. Fortin M, Stewart M, Poitras ME, et al. A systematic review of prevalence studies on multimorbidity: toward a more uniform methodology. Ann Fam Med 2012;10:142-51.

10. Garin N, Olaya B, Perales J, et al. Multimorbidity patterns in a national representative sample of the Spanish adult population PLOS ONE 2014;9:e84794

11. van Oostrom SH, Picavet HS, van Gelder BM, et al. Multimorbidity and comorbidity in the Dutch population-data from general practices. BMC Public Health 2012;12:715.

12. Taylor AW, Price K, Gill TK, et al. Multimorbidity-not just an older person's issue. Results from an Australian biomedical study. BMC Public Health 2010;10:718.

13. Nimako BA, Baiden F, Sackey SO, et al. Multimorbidity of chronic diseases among adult patients presenting to an inner-city clinic in Ghana. Glob Health 2013;9:61.

14. de Souza Santos Machado V, Valadares AL, da Costa-Paiva LS, et al. Multimorbidity and associated factors in Brazilian women aged 40 to 65 years: a population-based study. Menopause 2012;19:569-75.

15. Alaba O, Chola L. The social determinants of multimorbidity in South Africa. Int J Equity Health 2013;12:63.

16. Bhojani U, Beerenahalli TS, Devadasan R, et al. No longer diseases of the wealthy: prevalence and health-seeking for self-reported chronic conditions among urban poor in Southern India. BMC Health Serv Res 2013;13:306.

17. Bhojani U, Thriveni B, Devadasan R, et al. Out-of-pocket healthcare payments on chronic conditions impoverish urban poor in Bangalore, India. BMC Public Health 2012;12:990.

18. World Bank. South Asia-Countries [Internet]. South Asian Countries. (cited 31 July 2014). http://web.worldbank.org/WBSITE/ EXTERNAL/COUNTRIES/SOUTHASIAEXT/0, menuPK:158850 pagePK:146748 piPK:146812 theSitePK:223547,00.html

19. von Elm E, Altman DG, Egger M, et al. The Strengthening the Reporting of Observational Studies in Epidemiology (STROBE) Statement: Guidelines for reporting observational studies. Int J Surg 2014;12:1495-9.

20. Van Minh $\mathrm{H}, \mathrm{Ng} \mathrm{N}$, Juvekar S, et al. Self-reported prevalence of chronic diseases and their relation to selected sociodemographic variables: a study in INDEPTH Asian sites, 2005. Prev Chronic Dis 2008;5:A86.http://www.ncbi.nlm.nih.gov/pmc/articles/PMC2483549/

21. Arokiasamy $P$, Uttamacharya, Jain K. Multi-Morbidity, Functional Limitations, and Self-Rated Health Among Older Adults in India. SAGE Open 2015;5:2158244015571640.

22. Arokiasamy P, Uttamacharya U, Jain K. Multiple Chronic Diseases and Their Linkages with Functional health and Subjective Wellbeing among adults in the low-middle income countries: An Analysis of
SAGE Wave1 Data, 2007/10 [Internet]. International Institute for population Sciences; (cited 5 May 2015). 2013. http://mpra.ub. uni-muenchen.de/54914/

23. Pati S, Agrawal S, Swain S, et al. Non communicable disease multimorbidity and associated health care utilization and expenditures in India: cross-sectional study. BMC Health Serv Res 2014; $14: 451$.

24. Purty AJ, Bazroy J, Kar M, et al. Morbidity pattern among the elderly population in the rural area of Tamil Nadu, India. Turk J Med Sci 2006;36:45.

25. Khanam MA, Streatfield PK, Kabir ZN, et al. Prevalence and patterns of multimorbidity among elderly people in rural Bangladesh: a cross-sectional study. J Health Popul Nutr 2011;29:406-14.

26. Chakrabarty D, Mandal PK, Manna N, et al. Functional Disability and Associated Chronic Conditions among Geriatric Populations in a Rural Community of India. Ghana Med J 2010;44:150-4.

27. Chakraborty S. Health seeking behavior of aged population of a rura blosk in West Bengal. [Internet]. St. Joseph's Press for Achutha Menon Centre for Health Science Studies; 2005 (cited 5 May 2015). http://dspace.sctimst.ac.in/jspui/bitstream/123456789/2075/1/MPH_ 2004_04.pdf

28. Banjare P, Pradhan J. Socio-economic inequalities in the prevalence of multi-morbidity among the rural elderly in Bargarh District of Odisha (India). PLOS ONE 2014:9:e97832.

29. Vadrevu L, Kumar V, Kanjilal B. Gender Differentials in the Impact of Multi-Morbidity on Self Rated Health in Rural West Bengal in India. IOSR Journal of Nursing and Health Science (IOSR-JNHS) [Internet]. Mar-Apr (cited 5 May 2015);4(2). http://iosrjournals.org/ iosr-jnhs/papers/vol4-issue2/Version-3/C04231621.pdf

30. Swami H, Bhatia V, Dutt R, et al. A Community Based Study of Morbidity Profile among Elderly in India. Bahrain Medical Bulletin 2002;24:13-6.

31. Gupta KL, Joshi K, Bhat A, et al. Mucormycosis of the transplanted kidney with renal papillary necrosis. Exp Clin Transplant 2013:11:554-7.

32. Joshi K, Kumar R, Avasthi A. Morbidity profile and its relationship with disability and psychological distress among elderly people in Northern India. Int J Epidemiol 2003;32:978-87.

33. Patel V, Chatterji S, Chisholm D, et al. Chronic diseases and injuries in India. Lancet 2011;377:413-28.

34. Caughey GE, Vitry Al, Gilbert AL, et al. Prevalence of comorbidity of chronic diseases in Australia. BMC Public Health 2008;8:221.

35. Prados-Torres A, Calderón-Larrañaga A, Hancco-Saavedra J, et al. Multimorbidity patterns: a systematic review. J Clin Epidemiol 2014:67:254-66.

36. Huntley AL, Johnson R, Purdy S, et al. Measures of multimorbidity and morbidity burden for use in primary care and community settings: a systematic review and guide. Ann Fam Med 2012;10:134-41.

37. Glynn LG, Valderas JM, Healy $P$, et al. The prevalence of multimorbidity in primary care and its effect on health care utilization and cost. Fam Pract 2011;28:516-23.

38. Fortin M, Lapointe L, Hudon C, et al. Multimorbidity and quality of life in primary care: a systematic review. Health Qual Life Outcomes 2004;2:51 